\title{
Angiotensin II type 2 receptor-interacting protein 3a suppresses proliferation, migration and invasion in tongue squamous cell carcinoma via the extracellular signal-regulated kinase-Snai2 pathway
}

\author{
TINGTING ZHAO $^{1 *}$, QIANTING HE $^{1 *}$, ZHONGHUA LIU $^{1}$, XUEQIANG DING ${ }^{1}$, \\ XIAOFENG ZHOU ${ }^{2,3}$ and ANXUN WANG ${ }^{1}$ \\ ${ }^{1}$ Department of Oral and Maxillofacial Surgery, The First Affiliated Hospital of Sun Yat-Sen University, Guangzhou, \\ Guangdong 501180, P.R. China; ${ }^{2}$ Center for Molecular Biology of Oral Diseases; ${ }^{3}$ Department of Periodontics, \\ College of Dentistry, University of Illinois at Chicago, Chicago, IL 60612, USA
}

Received June 27, 2014; Accepted September 9, 2015

DOI: $10.3892 / \mathrm{ol} .2015 .3898$

\begin{abstract}
Our previous studies demonstrated that the downregulation of microtubule-associated tumor suppressor 1/angiotensin II type 2 receptor-interacting protein (MTUS1/ATIP) is associated with poor differentiation and prognosis in tongue squamous cell carcinoma (TSCC), and that ATIP1 exerts an antiproliferative effect on TSCC. The aim of the present study was to further investigate the anticancer effect of MTUS1/ATIP3a in TSCC. It was observed that UM1 cells (a TSCC cell line with high migration and invasion ability) exhibited lower expression of ATIP3a compared with UM2 cells (a TSCC cell line with lower migration and invasion ability). Restoration of ATIP3a expression in UM1 cells exerted antiproliferative effects and inhibited migration and invasion, whereas knockdown of ATIP3a promoted proliferation, migration and invasion in UM2 cells. Restoration of ATIP3a expression inhibited the phosphorylation of extracellular signal-regulated kinase 1/2 (ERK1/2) and the expression of Snai2 and vimentin in UM1 cells, whereas knockdown of ATIP3a promoted the phosphorylation of ERK1/2 and the expression of Snai2 and vimentin in UM2 cells. Therefore, MTUS1/ATIP3a was found to suppress the proliferation, migration and invasion of TSCC cells via the ERK1/2-Snai2 pathway.
\end{abstract}

Correspondence to: Professor Anxun Wang, Department of Oral and Maxillofacial Surgery, The First Affiliated Hospital, Sun-Yat-Sen University, 58 Zhongshan Road II, Guangzhou, Guangdong 510080, P.R. China

E-mail:wang_anxun@aliyun.com

*Contributed equally

Key words: microtubule-associated tumor suppressor 1, angiotensin II type 2 receptor-interacting protein $3 \mathrm{a}$, tongue squamous cell carcinoma, proliferation, migration, invasion

\section{Introduction}

Tongue squamous cell carcinoma (TSCC) is significantly more aggressive compared with other forms of oral cancer, with a propensity for rapid local invasion and spread, and a high recurrence rate (1). TSCC is characterized by genetic instabilities, including frequent loss of heterozygosity $(\mathrm{LOH})$ at the chromosomal region 8p21.3-p22 (2). Microtubule-associated tumor suppressor gene (MTUS1) is one of the candidate tumor suppressor genes that reside in this chromosomal region. In our previous study using single-nucleotide polymorphism (SNP) array-based $\mathrm{LOH}$ profiling on a large panel of oral cancer cell lines, a frequent $\mathrm{LOH}$ region was identified at 8 p22-p21.3, a region containing the MTUS1 gene, which was initially identified as a potential tumor suppressor gene in pancreatic cancer (2).

MTUS1 has been identified as an 8p22 candidate tumor suppressor gene encoding a family of angiotensin II type 2 (AT2) receptor-interacting proteins (ATIPs). Alternative exon utilization of this gene leads to five known transcript variants that code for five different protein isoforms, namely ATIP1, ATIP2, ATIP3a, ATIP3b and ATIP4 $(3,4)$. The ATIP polypeptides exhibit distinct motifs in the amino-terminus for localization to the cytosol, nucleus or cell membrane (ATIP1, ATIP3 and ATIP4, respectively), suggesting that MTUS1 gene products may be involved in a variety of intracellular functions in an AT2-dependent and -independent manner $(3,4)$.

The downregulation of the MTUS1 gene has been documented in a number of cancer types (5-10); among the five isoforms, ATIP1 and ATIP3a have been found to have a tumor suppressor function (6,7). Xiao et al (11) found that MTUS1 expression was correlated with tumor grade, stage, size and number in bladder cancer, and patients with low levels of MTUS1 mRNA expression had a poor prognosis compared with those exhibiting high MTUS1 expression. Our previous study also suggested that downregulation of MTUS1/ATIP was a frequent event in TSCC and premalignant lesions, e.g., leukoplakia. The downregulation of MTUS1/ATIP was also correlated with poor 
differentiation and reduced overall survival $(12,13)$. ATIP1 and ATIP3a were found to be the major isoforms produced by the MTUS1 gene in epithelial cells of the tongue and were significantly downregulated in TSCC tissues (13). Restoration of ATIP1 expression led to G1 arrest, apoptosis and reduced cell proliferation in TSCC cell lines (13).

The present study further investigated the role of ATIP3a in TSCC. First, the antiproliferative effect of ATIP3a in TSCC was observed. Subsequently, the role of ATIP3a in the migration and invasion of TSCC cells and the involvement of extracellular signal-regulated kinase 1/2 (ERK1/2)-Snai2 signaling induced by ATIP3a was investigated. The aim of this study was to elucidate the role of MTUS1/ATIP3a in the suppression of proliferation, migration and invasion of TSCC cells via the ERK1/2-Snai2 pathway. The current study was approved by the Medical Ethics Committee of the First Affiliated Hospital of Sun Yat-Sen University (Guangzhou, China).

\section{Materials and methods}

Cell culture and transfections. Human TSCC cell lines (UM1 and UM2) were provided by Dr. Tomohiro Matsumura (Department of Oral and Maxillofacial Surgery II, Okayama University Dental School, Okayama, Japan). The cells were maintained in Dulbecco's modified Eagle's medium/F12 (Gibco; Thermo Fisher Scientific, Grand Island, NY, USA) containing 10\% fetal bovine serum, 1,000 U/ml penicillin and $500 \mu \mathrm{g} / \mathrm{ml}$ streptomycin [all obtained from (Gibco) Thermo Fisher Scientific] in a $37^{\circ} \mathrm{C}$ incubator with $5 \% \mathrm{CO}_{2}$. UM1 and $\mathrm{UM} 2$ are paired cell lines from a single TSCC patient, with UM1 being more aggressive compared with UM2 in terms of cell migration and invasion (14). The expression vector containing the coding sequence of human ATIP3a was a gift from Dr. Clara (Cochin Institute, University Paris Descartes, Paris, France) (7). For functional analyses, the ATIP3a expression vector or empty vector (pCDNA3; Invitrogen; Thermo Fisher Scientific), gene-specific small interfering RNA (siRNA) for ATIP3a and control non-targeting siRNA (GenePharma Co., Ltd., Shanghai, China) were transfected into the appropriate cells using Lipofectamine ${ }^{\circledR}$ Transfection Reagent (Invitrogen; Thermo Fisher Scientific) according to the manufacturer's protocol. The three sequences of the ATIP3a siRNA are presented in Table I.

Cell proliferation assays. Proliferation was measured using an MTT assay, as previously described (13). In brief, the cells were seeded in 96-well plates at a density of $5 \times 10^{3}$ cells per well. Cell proliferation was analyzed after 24 or $48 \mathrm{~h}$ by incubating the cells with $1 \mathrm{mg} / \mathrm{ml}$ MTT tetrazolium salt (Sigma-Aldrich, St. Louis, MO, USA). Absorbance (A) at $570 \mathrm{~nm}$ was measured and the cell inhibition rate was calculated as follows: $\left(1-\mathrm{A}_{\text {treated }} / \mathrm{A}_{\text {control }}\right) \times 100 \%$.

Cell migration assay. Cell migration was measured using a Transwell ${ }^{\circledR}$ assay as previously described (14) with BD BioCoat Control Cell Culture Inserts containing an 8.0- $\mu \mathrm{m}$ polyethylene terephthalate membrane without matrix (BD Biosciences, Bedford, MA, USA). In brief, the cells were seeded in the upper chamber of the Transwell ${ }^{\circledR}$ plates. After $24 \mathrm{~h}$, the cells on the lower surface of the membrane were fixed and stained with DAPI solution in the dark. Three random fields were captured
Table I. Sequences of siRNA used for transfection.

\begin{tabular}{lc}
\hline Type of siRNA & Sequence \\
\hline ATIP3a siRNA & \\
No. 1 & \\
Sense & 5'-GGGUAAUCGAGGGCUUAAATT-3' \\
Antisense & 5'-UUUAAGCCCUCCAUUACCCTT-3' \\
No. 2 & \\
Sense & 5'-GCCCAAGACAUGACUUACATT-3' \\
Antisense & 5'-UGUAAGUCAUGUCUUGGGCTT-3' \\
No. 3 & \\
Sense & 5'-GGUGUUAGAUAUGCAUAAATT-3' \\
Antisense & 5'-UUUAUGCAUAUCUAACACCTT-3' \\
Control siRNA & \\
Sense & 5'-UUCUUCGAACGUGUCACGUTT-3' \\
Antisense & 5'-ACGUGACACGUUCGGAGAATT-3'
\end{tabular}

ATIP3a, angiotensin II type 2 receptor-interacting protein 3a; siRNA, small interfering RNA.

under a light microscope (magnification, x20; DMI3000B; Leica, Wetzlar, Germany) and the number of cells on the bottom surface was compared between the groups.

Cell invasion assay. Cell invasion was measured by the Transwell $^{\circledR}$ assay as previously described (14), using BD BioCoat BD Matrigel $^{\mathrm{TM}}$ Invasion Chamber containing a layer of $\mathrm{BD}$ Matrigel $^{\mathrm{TM}}$ Basement Membrane Matrix (BD Biosciences). In brief, the cells were seeded in the upper Boyden chambers of the cell culture inserts. After $24 \mathrm{~h}$ of incubation, cells remaining in the upper chamber or on the upper membrane were carefully removed. Cells adhering to the lower membrane were stained with DAPI solution. Three random fields were captured under a light microscope (magnification, x20; DMI3000B; Leica) and the number of cells on the bottom surface was compared between the groups.

Western blot analysis. Western blot analysis was performed to detect the levels of ERK1/2 [mouse anti-human monoclonal antibody (mAb); 1:1,000; cat. no. 4396], phosphorylated-ERK1/2 (p-ERK1/2; rabbit anti-human mAb; 1:1,000; cat. no. 4376), Snai2 (rabbit anti-human mAb; 1,000; cat. no. 9858), vimentin (mouse anti-human mAb; 1:1,000; cat. no. 3390), E-cadherin (rabbit anti-human mAb; 1,000; cat. no. 3195), GAPDH (rabbit anti-human mAb; 1:1,000; cat. no. 5174) (all from Cell Signaling Technology, Beverly, MA, USA), and MTUS1 (mouse anti-human mAb; 1:1,000; cat. no. H00057509-M01; Abnova, Taipei, Taiwan) in TSCC cells. The cells were lysed with a radioimmunoprecipitation assay lysis buffer (Santa Cruz Biotechnology, Inc., Santa Cruz, CA, USA). Aliquots (30-50 $\mu \mathrm{g}$ ) of cellular proteins were resolved by $10 \%$ SDS-PAGE, electrotransferred onto polyvinylidene difluoride membranes and immunoprobed. The protein/antibody complexes were detected by chemiluminescence (CSPD chemiluminescent substrate; Tropix, Bedford, MA, USA) according to the manufacturer's protocol. 

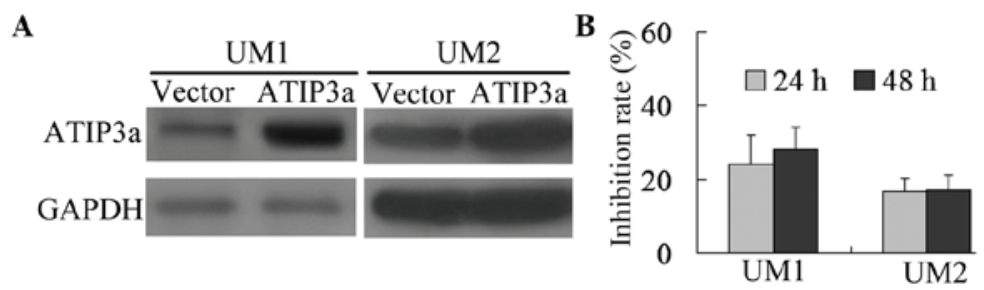

Figure 1. ATIP3a inhibited the proliferation of tongue squamous cell carcinoma cells. (A) Expression of ATIP3a in UM1 and UM2 cells following transfection was detected by western blot analysis with GAPDH used as the loading control. (B) ATIP3a overexpression was found to significantly inhibit the proliferation of TSCC cells $(\mathrm{P}<0.05)$. ATIP3a, angiotensin II type 2 receptor-interacting protein $3 \mathrm{a}$.

The same blots were used for analysis of the loading control, GAPDH, following stripping of the membrane.

Statistical analysis. All the experiments were performed in triplicate, and the data are presented as means \pm standard deviation. Student's t-tests were used to compare the differences between the groups. For all the statistical analyses, $\mathrm{P}<0.05$ was considered to indicate statistically significant differences.

\section{Results}

ATIP3a inhibits the proliferation of TSCC cells. The MTT assay was employed to evaluate the antiproliferative effect of ATIP3a in TSCC. As shown in Fig. 1A, ATIP3a was overexpressed in UM1 and UM2 cells following transfection. When UM1 or UM2 cells were transfected with ATIP3a expression vector, a statistically significant inhibition in cell proliferation was observed when compared with the cells transfected with the empty vector (Fig. 1B). The inhibition rate of ATIP3a was $24.1 \%(24 \mathrm{~h})$ and $28.3 \%$ (48 h) in UM1 cells, and $~ 16.9 \%$ (24 h) and $17.1 \%$ (48 h) in UM2 cells. The antiproliferative effect of ATIP3a did not differ between 24 and $48 \mathrm{~h}$ of transfection. These results suggest that ATIP3a possesses significant antiproliferative activity against TSCC.

ATIP3a is associated with inhibition of migration and invasion of TSCC cells. As shown in Fig. 2A, the protein levels of ATIP3a in UM2 cells were higher compared with those of UM1 cells, which normally have a higher migration and invasion ability compared with UM2 cells $(14,15)$. To evaluate the association between ATIP3a and the migration and invasion ability of TSCC cells, UM1 cells were first transfected with ATIP3a. As shown in Fig. 2B and C, the migration and invasion ability of the UM1 cells was significantly inhibited following transfection with ATIP3a. ATIP3a was subsequently knocked down by RNA interference in UM2 cells. As shown in Fig. 3A, the protein level of ATIP3a in UM2 cells was significantly decreased following transfection with ATIP3a siRNA. Among the three sequences of ATIP3a siRNA, sequence no. 1 exhibited the greatest silencing effect (Fig. 3A); therefore this sequence was selected for the following experiment. UM2 cells transfected with ATIP3a siRNA displayed significantly increased migration and invasion ability compared with the control siRNA-transfected UM2 cells (Fig. 3B and C). Furthermore, it was observed that ATIP3a siRNA promoted the proliferation of UM2 cells (Fig. 3D). These results indicate that ATIP3a is associated with inhibition of migration and invasion of TSCC cells.
ATIP3a regulates the ERK-Snai2 pathway in TSCC cells. As shown in Fig. 4A, the ectopic expression of ATIP3a in UM1 cells led to an increase in ERK1/2 and E-cadherin and a notable decrease in p-ERK1/2, Snai2 and vimentin. However, following knockdown of ATIP3a in UM2 cells, the protein levels of p-ERK1/2, Snai2 and vimentin were markedly increased and the protein levels of ERK1/2 and E-cadherin were markedly decreased (Fig. 4B). These results indicate that the ERK1/2-Snai2 pathway contributes to ATIP3a-induced inhibition of proliferation, migration and invasion of TSCC cells.

\section{Discussion}

MTUS1/ATIP is one of the candidate tumor suppressor genes located in the chromosomal region 8p22-p21.3, which was identified in our previous study as one of the most frequent LOH (87.9\%) sites in oral cancer (2). Numerous studies have demonstrated that the deregulation of MTUS1/ATIP is associated with several types of cancer $(5-11,13)$, including hepatocellular, bladder, breast, colon, prostate and head and neck cancer. Our previous studies also demonstrated that the reduction of MTUS1 expression was associated with the development and prognosis of TSCC $(2,12,13)$ and revealed that ATIP1 and ATIP3a were the major isoforms of MTUS1 and were significantly decreased in TSCC. Restoring ATIP1 expression in TSCC cell lines induced $\mathrm{G}_{1} / \mathrm{G}_{0}$ arrest and reduced cell proliferation.

ATIP3a polypeptides contain a nuclear localization signal in their amino-terminus and may not colocalize with the seven-transmembrane domain AT2 receptor inside the cell, suggesting AT2-independent roles for ATIP3 proteins in the majority of tissues. Rodrigues-Ferreira et al (7) found that restoring ATIP3 expression in breast cancer led to reduced cancer cell proliferation, clonogenicity and anchorage-independent growth and reduced the incidence and size of xenografts grown in vivo. Molina et al (16) also found that ATIP3a was associated with reduced cancer cell proliferation and metastasis. ATIP3a silencing promoted breast cancer cell migration, whereas ATIP3a expression significantly reduced cell motility and directionality. The present study also demonstrated that ATIP3a overexpression in TSCC cells significantly inhibited their proliferation and that ATIP3a overexpression suppressed the migration and invasion ability of UM1 cells, whereas knockdown of ATIP3a promoted the migration and invasion ability of UM2 cells. All these results indicate that ATIP3a plays an important role in the proliferation, migration and invasion of TSCC cells. 
A

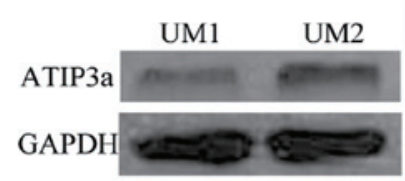

B

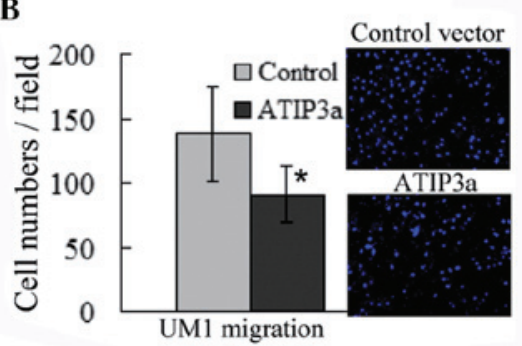

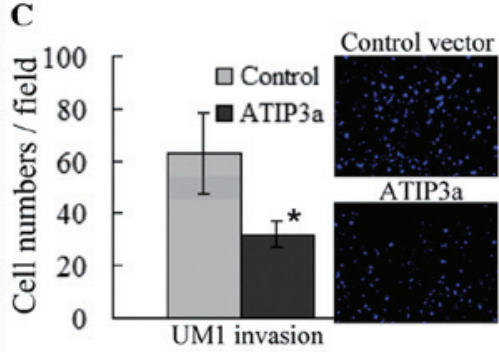

Figure 2. ATIP3a overexpression inhibited the migration and invasion ability of tongue squamous cell carcinoma cells. (A) Significantly higher expression levels of the ATIP3a protein were observed in UM1 cells compared with UM2 cells. ATIP3a overexpression significantly inhibited the (B) migration and (C) invasion of UM1 cells. "P<0.05. ATIP3a, angiotensin II type 2 receptor-interacting protein $3 \mathrm{a}$.

$\mathbf{A}$

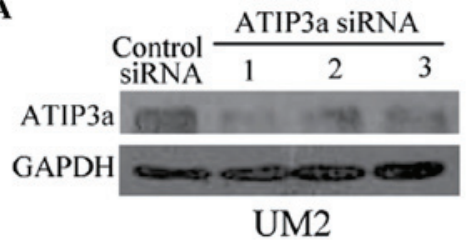

C

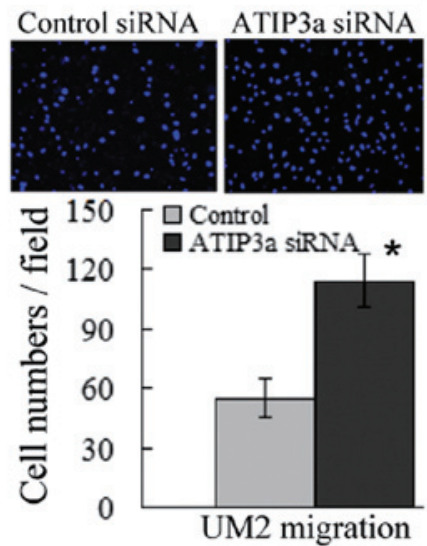

B

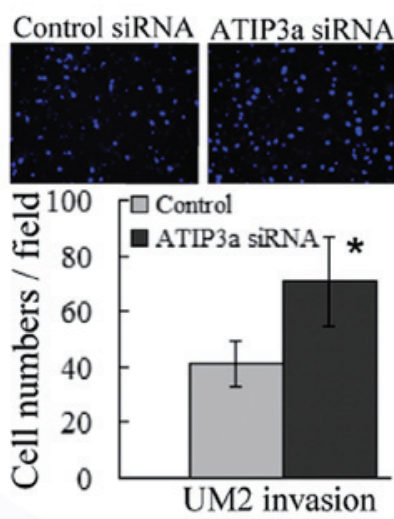

D

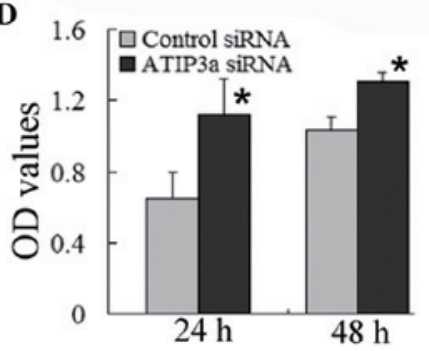

Figure 3. ATIP3a knockdown promoted the migration and invasion ability of tongue squamous cell carcinoma cells. (A) Significant reductions in ATIP3a protein levels were observed in the ATIP3a siRNA-transfected UM2 cells compared with the control siRNA-transfected cells. (B-D) ATIP3a knockdown promoted the (B) invasion, (C) migration and (D) proliferation ability of UM2 cells compared with that of the control siRNA-transfected UM2 cells. "P<0.05. ATIP3a, angiotensin II type 2 receptor-interacting protein 3a; siRNA, small interfering RNA; OD, optical density.
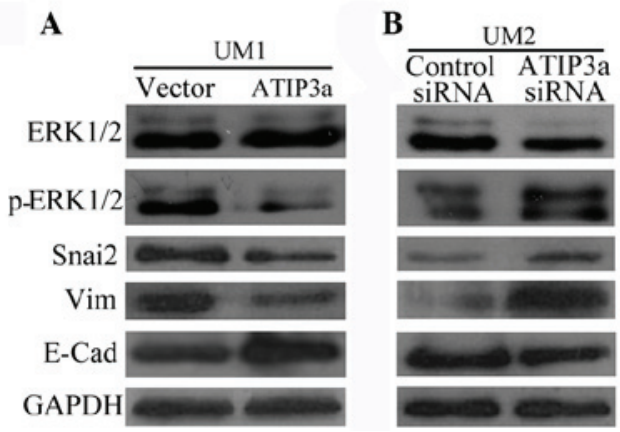

Figure 4. ATIP3a regulated the ERK-Snai2 pathway in tongue squamous cell carcinoma cells. (A) Differential expression of the ERK-Snai2 pathway in UM1 cells treated with either control vector or ATIP3a plasmid. (B) Differential expression of ERK-Snai2 pathway in UM2 cells treated with either control or ATIP3a siRNA. ATIP3a, angiotensin II type 2 receptor-interacting protein 3a; ERK, extracellular signal-regulated kinase; siRNA, small interfering RNA; Vim, vimentin; E-Cad, E-cadherin.
ATIP3a has been shown to be associated with the microtubule cytoskeleton and localizes at the centrosomes, mitotic spindle and intercellular bridge during cell division. ATIP3a expression alters the progression of cell division by promoting prolonged metaphase, thereby leading to a reduced number of cells undergoing active mitosis (7). To date, the knowledge of ATIP-regulated molecular pathways is relatively limited. The ERK signaling pathway has been found to play a crucial role in almost all cell functions (17). ERK2/ERK1 are two isoforms of ERK that belong to the family of mitogen-activated protein kinases (MAPKs). ATIP1 was previously shown to be involved in the inhibition of the ERK pathway $(6,13,18,19)$. The present study also demonstrated that ATIP3a is involved in the inhibition of ERK1/2 activity. The protein level of p-ERK1/2 was found to be significantly reduced in UM1 cells following transfection with ATIP3a, and increased in ATIP3a siRNA-transfected UM2 cells. 
It was recently reported that hundreds of proteins are under ERK-dependent control (20). Snai2 belongs to the Snail family of zinc-finger transcription factors, and is a well-established downstream target of the MAPK/ERK pathway in a number of cell types $(21,22)$. Our previous study (23) demonstrated that the MAPK-Snai2 pathway played an important role in salivary adenoid cystic carcinoma (SACC) metastasis. Snai2 is a downstream target of MAPK1 (ERK2); siRNA-mediated ERK2-knockdown suppressed Snai2 gene promoter activity and reduced the Snai2 protein level in SACC cells (23). Snail family genes are also best known for their role in epithelial-to-mesenchymal transition (EMT) (24). In several types of human cancer, there is an inverse association between E-cadherin and Snail gene family expression $(22,25,26)$. Wang et al (27) confirmed that Snai2 overexpression was correlated with reduced E-cadherin expression and enhanced vimentin expression in two independent cohorts of TSCC patients. In vitro, knockdown of Snai2 suppressed cell invasion and migration; by contrast, ectopic transfection of Snai2 led to enhanced cell invasion and migration (27). Our previous studies $(14,15)$ also revealed that $\mathrm{p}$-ERK1/2, Snai2 and vimentin exhibited higher expression levels and E-cadherin lower expression levels in UM1 cells compared with UM2 cells. The present study demonstrated that the overexpression of ATIP3a in UM1 cells led to an increase in ERK1/2 and E-cadherin and a notable decrease in $\mathrm{p}$-ERK1/2, Snai2 and vimentin. However, following ATIP3a knockdown in UM2 cells, the protein levels of p-ERK1/2, Snai2 and vimentin were markedly increased, whereas the protein levels of ERK1/2 and E-cadherin were markedly decreased. These results indicate that ERK1/2-Snai2 signaling contributes to ATIP3a-induced suppression of proliferation, migration and invasion of TSCC cells.

In conclusion, MTUS1/ATIP3a exerted a notable antiproliferative effect and inhibited the migration and invasion of TSCC cells via regulation of the ERK-Snai2 pathway. Thus, MTUS1/ATIP3a may be a novel therapeutic target for TSCC.

\section{Acknowledgements}

This study was supported in part by grants from the National Natural Science Foundation of China (nos. NSFC81072228 and NSFC81272953), the Guangdong Natural Science Foundation (no. S2011020002325), the research fund for the doctoral program of the Ministry of Education (no. 20120171110050), the Fundamental Research Funds for the Central Universities (no. 11ykzd09), the Program for New Century Excellent Talents in University (no. NCET-10-0857), the Project of Science and Technology of Guangdong Province (no. 2012B031800080) and the National Institutes of Health PHS (no. CA139596).

\section{References}

1. Wang A, Liu X, Sheng S, et al: Dysregulation of heat shock protein 27 expression in oral tongue squamous cell carcinoma. BMC Cancer 9: 167, 2009.

2. Ye H, Pungpravat N, Huang BL, et al: Genomic assessments of the frequent loss of heterozygosity region on 8p21.3-p22 in head and neck squamous cell carcinoma. Cancer Genet Cytogenet 176: 100-106, 2007.

3. Di Benedetto M, Bièche I, Deshayes F, et al: Structural organization and expression of human MTUS1, a candidate 8p22 tumor suppressor gene encoding a family of angiotensin II AT2 receptor-interacting proteins, ATIP. Gene 380: 127-136, 2006.
4. Yu J, Liu X, Ye H and Zhou X: Genomic characterization of the human mitochondrial tumor suppressor gene 1 (MTUS1): 5 ' cloning and preliminary analysis of the multiple gene promoters. BMC Res Notes 2: 109, 2009.

5. Di Benedetto M, Pineau P, Nouet S, et al: Mutation analysis of the 8p22 candidate tumor suppressor gene ATIP/MTUS1 in hepatocellular carcinoma. Mol Cell Endocrinol 252: 207-215, 2006.

6. Seibold S, Rudroff C, Weber M, Galle J, Wanner C and Marx M: Identification of a new tumor suppressor gene located at chromosome 8p21.3-22. FASEB J 17: 1180-1182, 2003.

7. Rodrigues-Ferreira S, Di Tommaso A, Dimitrov A, et al: 8p22 MTUS1 gene product ATIP3 is a novel anti-mitotic protein underexpressed in invasive breast carcinoma of poor prognosis. PLoS One 4: e7239, 2009.

8. Zuern C, Heimrich J, Kaufmann R, et al: Down-regulation of MTUS1 in human colon tumors. Oncol Rep 23: 183-189, 2010.

9. Louis SN, Chow L, Rezmann L, et al: Expression and function of ATIP/MTUS1 in human prostate cancer cell lines. Prostate 70: 1563-1574, 2010.

10. Bacolod MD and Barany F: Gene dysregulations driven by somatic copy number aberrations - biological and clinical implications in colon tumors: A paper from the 2009 William Beaumont Hospital Symposium on Molecular Pathology. J Mol Diagn 12: 552-561, 2010.

11. Xiao J, Chen JX, Zhu YP, Zhou LY, Shu QA and Chen LW: Reduced expression of MTUS1 mRNA is correlated with poor prognosis in bladder cancer. Oncol Lett 4: 113-118, 2012.

12. Zhou X, Temam S, Oh M, et al: Global expression-based classification of lymph node metastasis and extracapsular spread of oral tongue squamous cell carcinoma. Neoplasia 8: 925-932, 2006.

13. Ding X, Zhang N, Cai Y, et al: Down-regulation of tumor suppressor MTUS1/ATIP is associated with enhanced proliferation, poor differentiation and poor prognosis in oral tongue squamous cell carcinoma. Mol Oncol 6: 73-80, 2012.

14. Liu Z,Li S, Cai Y, et al: Manganese superoxide dismutase induces migration and invasion of tongue squamous cell carcinoma via $\mathrm{H}_{2} \mathrm{O}_{2}$-dependent Snail signaling. Free Radic Biol Med 53: 44-50, 2012.

15. Zhou Z, Zheng C, Li S, et al: AFM nanoindentation detection of the elastic modulus of tongue squamous carcinoma cells with different metastatic potentials. Nanomedicine 9: 864-874, 2013.

16. Molina A, Velot L, Ghouinem L, et al: ATIP3, a novel prognostic marker of breast cancer patient survival, limits cancer cell migration and slows metastatic progression by regulating microtubule dynamics. Cancer Res 73: 2905-2915, 2013.

17. Deschênes-Simard X, Kottakis F, Meloche S and Ferbeyre G: ERKs in cancer: friends or foes? Cancer Res 74: 412-419, 2014.

18. Nouet S, Amzallag N, Li JM, et al: Trans-inactivation of receptor tyrosine kinases by novel angiotensin II AT2 receptor-interacting protein, ATIP. J Biol Chem 279: 28989-28997, 2004.

19. Wruck CJ, Funke-Kaiser H, Pufe T, et al: Regulation of transport of the angiotensin AT2 receptor by a novel membrane-associated Golgi protein. Arterioscler Thromb Vasc Biol 25: 57-64, 2005.

20. von Kriegsheim A, Baiocchi D, Birtwistle M, et al: Cell fate decisions are specified by the dynamic ERK interactome. Nat Cell Biol 11: 1458-1464, 2009.

21. Chen H, Zhu G, Li Y, et al: Extracellular signal-regulated kinase signaling pathway regulates breast cancer cell migration by maintaining slug expression. Cancer Res 69: 9228-9235, 2009.

22. Joseph MJ, Dangi-Garimella S, Shields MA, et al: Slug is a downstream mediator of transforming growth factor-betal-induced matrix metalloproteinase-9 expression and invasion of oral cancer cells. J Cell Biochem 108: 726-736, 2009.

23. He Q, Zhou X, Li S, et al: MicroRNA-181a suppresses salivary adenoid cystic carcinoma metastasis by targeting MAPK-Snai2 pathway. Biochim Biophys Acta 1830: 5258-5266, 2013.

24. Cobaleda C, Pérez-Caro M, Vicente-Dueñas C and Sánchez-García I: Function of the zinc-finger transcription factor SNAI2 in cancer and development. Annu Rev Genet 41: 41-61, 2007.

25. Hardy RG, Vicente-Dueñas C, González-Herrero I, et al: Snail family transcription factors are implicated in thyroid carcinogenesis. Am J Pathol 171: 1037-1046, 2007.

26. Al Saleh S, Sharaf LH and Luqmani YA: Signalling pathways involved in endocrine resistance in breast cancer and associations with epithelial to mesenchymal transition (Review). Int J Oncol 38: 1197-1217, 2011.

27. Wang C, Liu X, Huang H, et al: Deregulation of Snai2 is associated with metastasis and poor prognosis in tongue squamous cell carcinoma. Int J Cancer 130: 2249-2258, 2012. 